GBD method resulted in greater redistribution of X59 cases to transport injury.

\title{
0170 X59: REDISTRIBUTION USING LINKED DATA AND THE IMPACT ON YLL CALCULATIONS
}

B J Gabbe, ${ }^{*}$ R A Lyons Correspondence: Department of Medicine, Nursing and Health Sciences, School of Public Health and Preventive Medicine, Monash University, The Alfred Hospital Commercial Rd, Melbourne, Victoria 3004, Australia

\subsection{6/ip.2010.029215.170}

Background The ICD-10 X59 cause code (unspecified) is prevalent in mortality datasets. The GBD project proportionally redistributes X59 deaths by age and gender for calculation of years of life lost (YLL) but the validity of the method has been questioned.

Objectives To establish the prevalence of X59 coding of deaths and the impact on YLL estimates using different redistribution methods.

Methods Registered deaths in Wales (2003-2007) with an ICD-10 injury cause code were included. Data were linked to hospital admissions if the place of death was a hospital. Three redistribution methods were studied: (i) GBD project method, (ii) Proportional redistribution based on the nature of injury and (iii) Proportional redistribution based on the specified causes for X59 cases from linked data. YLLs were calculated for each method.

Results $24 \%$ ( $n=944)$ of unintentional deaths were coded as X59. 92\% involved deaths in people aged $70+$ years. Most involved proximal femur fractures and subdural haematomas. A linked admission was available for $99 \%$ of eligible cases $(n=797)$. The cause of death was specified for 546 cases. Falls were predominant.

Conclusions The proportion of YLLs attributed to cause categories across the methods was comparable because of the age discounting in the YLL formula. There were differences in the proportion of deaths attributed to cause categories with redistribution using the nature of injury or linked data resulting in the majority of X59 cases redistributed to falls, while the 